\title{
WAVELET TRANSFORM BASED DETECTION OF PHOTON-LIMITED AND LOW CONTRAST OBJECTS
}

\author{
James P. LeBlanc \\ Klipsch School of ECE \\ New Mexico State University \\ Las Cruces, NM USA
}

\author{
Mysore Raghuveer \\ Dept. of Elec. Eng. \\ Rochester Institute of Technology \\ Rochester, NY USA
}

\section{WAVELET TRANSFORM}

The continuous wavelet transform (CWT) of a function $f(t)$ is defined in [6] as

$$
\begin{aligned}
\operatorname{CWT}_{f}(a, b) & =\int_{-\infty} \infty \psi_{a, b}^{*}(t) f(t) d t \\
& =\frac{1}{\sqrt{a}}\left\langle\psi_{a, b}(t), f(t)\right\rangle,
\end{aligned}
$$

the use of the wavelet transform on the complex envelope of the signal of interest. This has the advantage of reducing "rippling" effects seen in the transform of the original waveform. An example of further post-processing on the wavelet-transformed data is provided.

\section{INTRODUCTION}

Photon-limited imaging is useful in low radiation level applications such as: night vision, astronomy, and sonar. The recording of such objects relies on counting or detecting photons as opposed to using intensity measurements as is done when there is sufficient illumination or radiation. Low radiation level objects (images) are modeled as a collection of Dirac delta functions,

$$
I(x, y)=\sum_{k=1}^{N} \delta\left(x-x_{k}, y-y_{k}\right)
$$

where $I(x, y)$ is the computed intensity at coordinates $(x, y)$ and $N$ is the number of photons in the vicinity. The photon generation is usually modeled as a Poisson process. Thus, the intensity is related to the photon arrival rate. The lower the photon rate, the more jagged the object appears, rendering detection and classification in the presence of observation noise more difficult.

\section{EXISTING METHODS OF PHOTON-LIMITED DETECTION}

There are several reported techniques for the detection, localization, and classification of photon-limited objects in additive noise. They tend to be either correlation-based [1], [2], or Fourier transform-based [3]. A shortcoming of such techniques is their inherent sensitivity to object scaling. Furthermore, some of them rely on multiple data snapshots for enhancing the SNR. Herein, we propose techniques based on the continuous wavelet transform (CWT) that take advantage of its ability to reject noise and to localize objects in space or time, as well as in scale. where $a \in \mathcal{R}^{+}$and $b \in \mathcal{R},{ }^{*}$ denotes the complex conjugate, and $\psi_{a, b}$ denotes,

$$
\psi_{a, b}=\frac{1}{\sqrt{a}} \psi\left(\frac{t-b}{a}\right)
$$

which represents shifted and scaled version of the "mother wavelet" function $\psi(t)$.

Thus, the CWT of a one-dimensional function is a twodimensional function of the parameters $a$ and $b$. The parameter $a$ is a scaling parameter, small values of $a$ may be associated with small scales (hence high frequencies). A time shift is represented by parameter $b$. In this light, we see the CWT of a signal provides a description of the signal both in terms of frequency components and temporal localization. It is this characteristic we wish to exploit here in the task of photon-limited imaging. For our purposes, we consider our original signal to be discrete in nature (i.e. sampled) and use the DWT, a discretized version of (2),

$$
\operatorname{DWT}_{f}(a, b)=\frac{1}{\sqrt{a}}\left\langle\psi\left(\frac{t-b}{2^{k}}\right), x(k)\right\rangle .
$$

It should be noted that there are limitless functions that may used as the "elementary function" in (2) or (4). In many situations, the choice of this "mother wavelet" is of little consequence. For the examples presented herein, we choose to use the "Mexican Hat Wavelet" [4] defined as,

$$
\psi=\frac{\left(1-t^{2}\right)}{2} \exp \left(-t^{2}\right)
$$

\subsection{Modeling a One-Dimensional Photon-Limited Object}

A photon-limited version of a one-dimensional object $O(x)$ is modeled as a collection of randomly positioned impulses

$$
O_{p}(x)=\sum_{i} \delta\left(x-x_{i}\right)
$$


On average, the density of the impulses in the neighborhood of $x_{0}$ is proportional to $O\left(x_{0}\right)$ if we assume photon generation to be a Poisson process with arrival rate proportional to object intensity. If $P(x)$ is the photon count in a small interval $\Delta x$ centered at $x$ then

$$
E\{P(x)\} \propto O(x) \Delta x .
$$

A good approximation to a realization of the above process is obtained by the following:

1. For any $x_{0}$ draw a random deviate from a uniform distribution between 0 and 1 .

2. Multiply the deviate by $O\left(x_{0}\right)$, producing the approximation to $O_{p}(x)$.

3. For a smoother function, that is, a higher photon rate, multiply the average of several independent uniform random deviates by $O\left(x_{0}\right)$.

\subsection{Detecting the Object}

The situation of interest here is in detecting and localizing $O(x)$ when we have a realization of

$$
D(x)=O_{p}(x)+n(x)
$$

where $D(x)$ is the observed process, $O_{p}(x)$ is the photonlimited object and $n(x)$ is additive noise. A common method of detection is the counting of photons in an observation window. This is equivalent to integrating $D(x)$ within a sliding window. If the count exceeds a threshold the data within the window is classified as part of the object; it is treated as background noise otherwise. This essentially amounts to cross correlating the data with the window and then applying the threshold. The window size affects the classification accuracy. A window that is too wide would require a high threshold to suppress false alarms thus reducing the probability of detection as well. The opposite is true for a narrow window. We propose the use of the wavelet transform precisely to take advantage of the fact that the transform offers correlation with windows of varying sizes due to the dilation that is inherent in the transform.

\section{USE OF WAVELET TRANSFORM PEAKS IN OBJECT DETECTION}

Figure 1, shows an example signal of interest, a square pulse of duration 20 and unit amplitude. Figure 2 shows an image of the magnitude of each element of the DWT $(a, b)$ matrix. The horizontal axis relates to the pulse position (wavelet translations), while the vertical axis relates to the pulse duration (wavelet scaling). The local maxima (peaks) of DWT are denoted on the image with a black "o" and a white "+". The smaller subplots show the position and magnitude of these peaks in both position and scale.

Regarding Figure 2, we see that there are ripples in the wavelet transform of the pulse as seen by the black/white bands in the image or the multiple peaks in position and scale. Such rippling may cause problems with further postprocessing of the wavelet transform.

\subsection{Use of Complex Envelope}

A proposed remedy introduced herein is to use the complex envelope of $O(x)$, denoted by $\widetilde{O}(x)$ and defined as,

$$
\widetilde{O}(x)=O(x)+j \widehat{O}(x)
$$

where $\widehat{O}(x)$ is the Hilbert transform of $O(x)$. Figure 3 depicts the wavelet transform of $\widetilde{O}(x)$. Note how the rippling has been reduced greatly. Such reduction provides benefits for the detection methods explored herein.

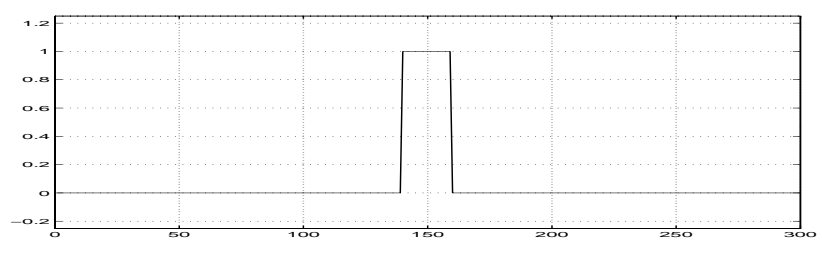

Figure 1. Example Object $O(x)$

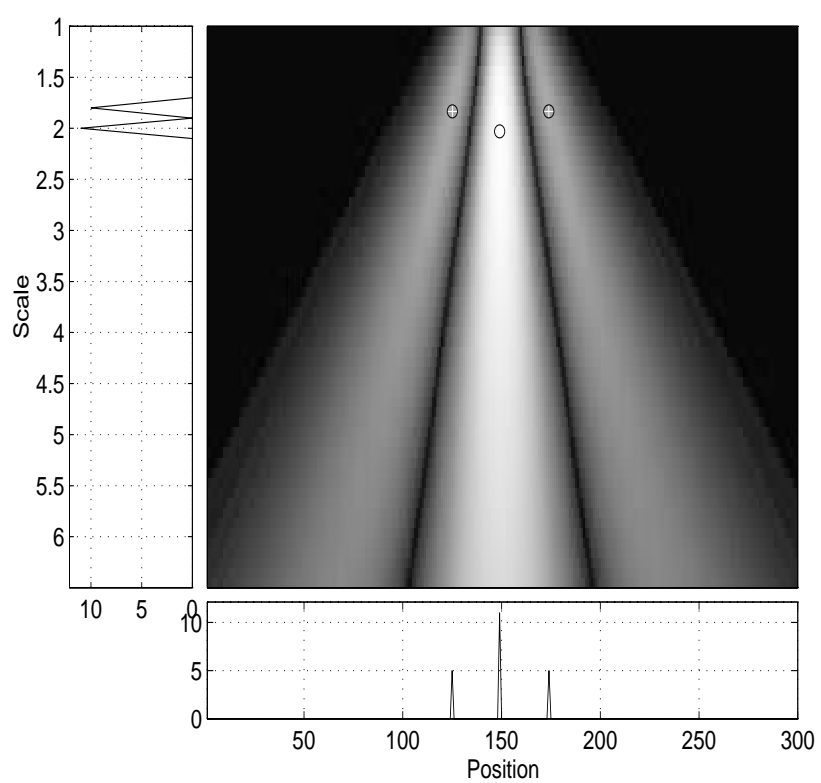

Figure 2. Wavelet Transform of $O(x)$

\section{DATA EXAMPLE}

Sample results are shown here for data consisting of two photon-limited objects separated in time and of differing

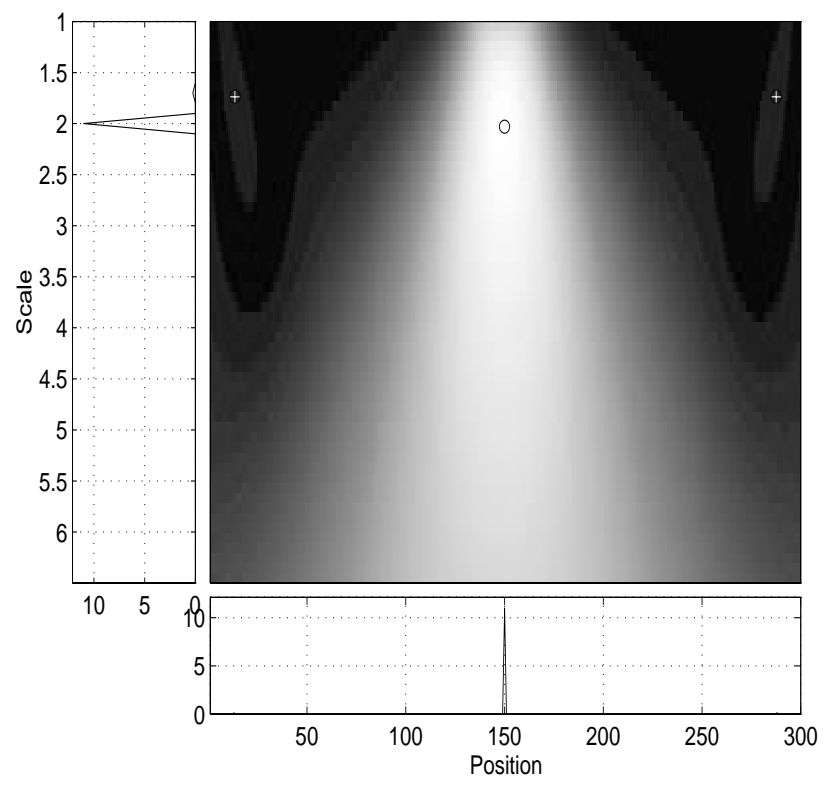

Figure 3. Wavelet Transform of $\widetilde{O}(x)$ 
size. Figure 4 shows the original objects $O(x)$, photonlimited objects $O_{p}(x)$, and the observed data $D(x)$ corresponding to the photon-limited objects in additive noise with $\sigma=0.08$. The approximate SNR (defined as the ratio of power of $O_{p}$ to power of $n(x)$ is $-3.9 \mathrm{~dB}$.
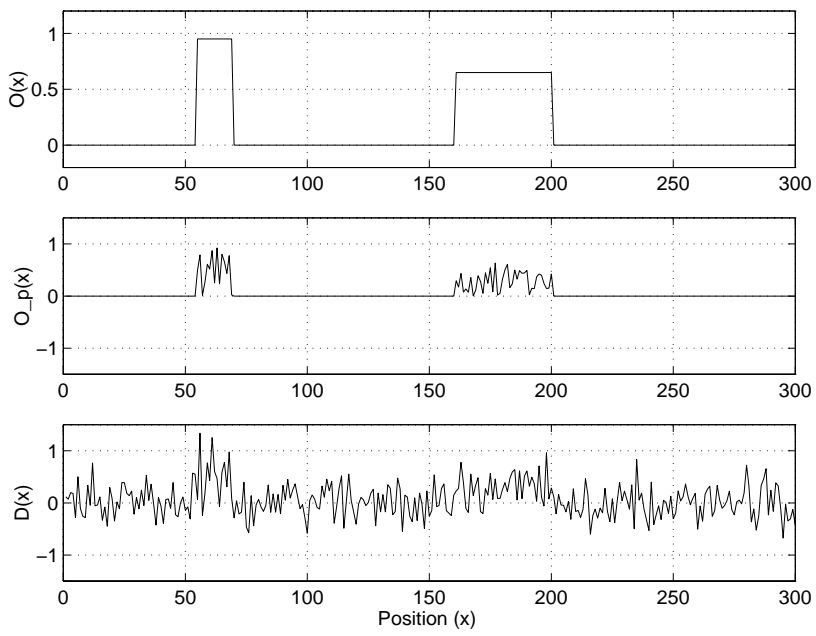

Figure 4. Objects $O(x)$, Photon-limited Objects $O_{P}(x)$, and Observed Data $D(x)$

Before looking at the effects of photon-limiting and observation noise we depict in Figure 5 the peaks of the wavelet transform when each of the single objects considered separately. That is, it plots the location of the peaks of DWT $\left(O_{i}(x)\right)$ where $O_{i}(x)$ is the signal due to the $i$ th object alone. Here, $O_{1}(x)$ is a pulse of width 15 starting at 55, and $\mathrm{O}_{2}(x)$ is a pulse of width 40 starting at 161 . Notice that the peaks placement in both time and scale agree with the actual object in Figure 4a. This would be the output of a "perfect" detector.

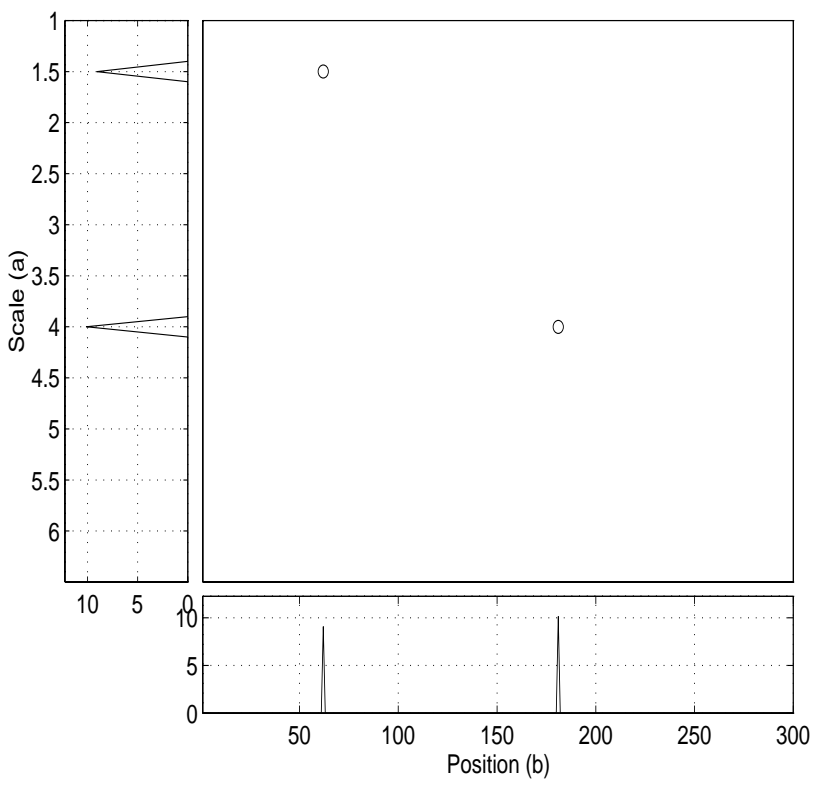

Figure 5. Peaks of DWT of Singleton Objects

Figure 6 depicts the wavelet transform and its associated peaks for the two objects considered together (i.e. from Figure 4a). Notice that the peaks' placement in time agree with the actual objects, but the scale term has been perturbed from the actual peak due to "interference" between the two objects. Such displacement may be small when the objects are well separated in time (relative to their scale). More troubling, however, is the appearance of multiple peaks (the six such peaks in the positional plots are due to only two objects). How is one to distinguish peaks due to actual objects and these peaks introduced by the DWT and the object interaction?

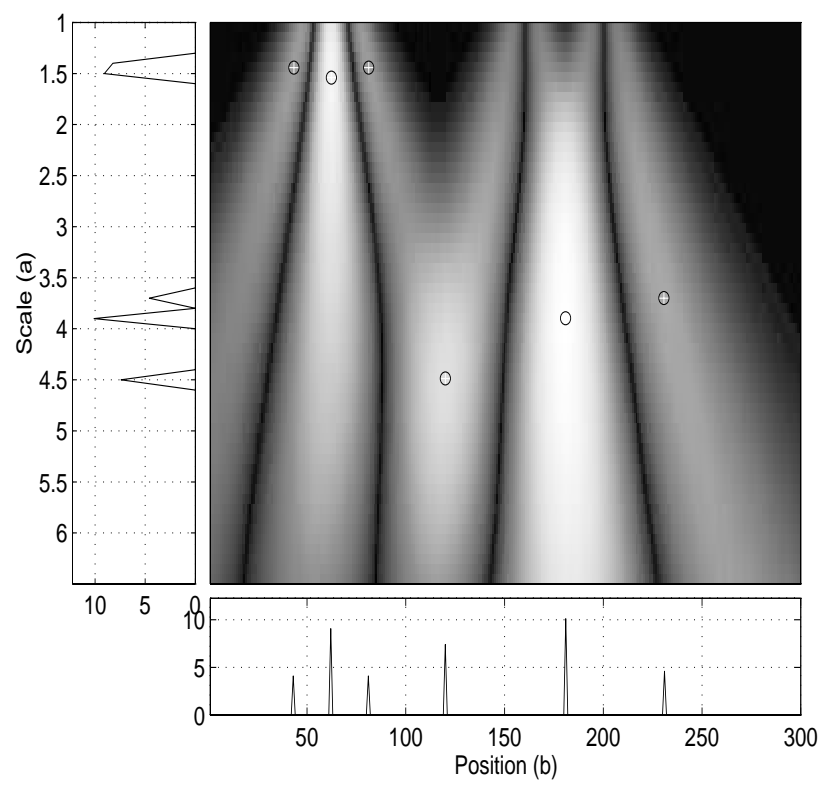

Figure 6. DWT of $O(x)$

Figure 7 demonstrates the effectiveness of the use of the complex envelope in reducing/removing such "false peaks". Here we have only two positional/scale peaks corresponding to the actual objects position/scale (albeit biased peaks).

Finally, we present the results of such processing of the photon-limited objects in measurement noise. Figure 8 shows the wavelet transform and associated peaks of $D(x)$. As in the noiseless case above, there exist multiple peaks in both position and scale axes making object detection and feature determination difficult.

In Figure 9. the wavelet transform of $\widetilde{O}(x)$ is computed and displayed with its associated peaks. The lack of multiple peaks makes scale and position identification of the object clearer.

Again, the use of the complex envelope (this time with photon limited and observation noise) demonstrates the effectiveness of removing the false peaks and maintaining those peaks actually due to objects as seen in

\section{REFERENCES}

[1] G. M. Morris, "Scene matching using photon limited images," J. Opt. Soc. Am. A, pp. 482-488, May 1984.

[2] X. Shi, R. K. Ward, "Noniterative image reconstruction from photon-limited data with the use of self-cross-correlation," J. Opt. Soc. Am. A, p. 47, Jan. 1995 


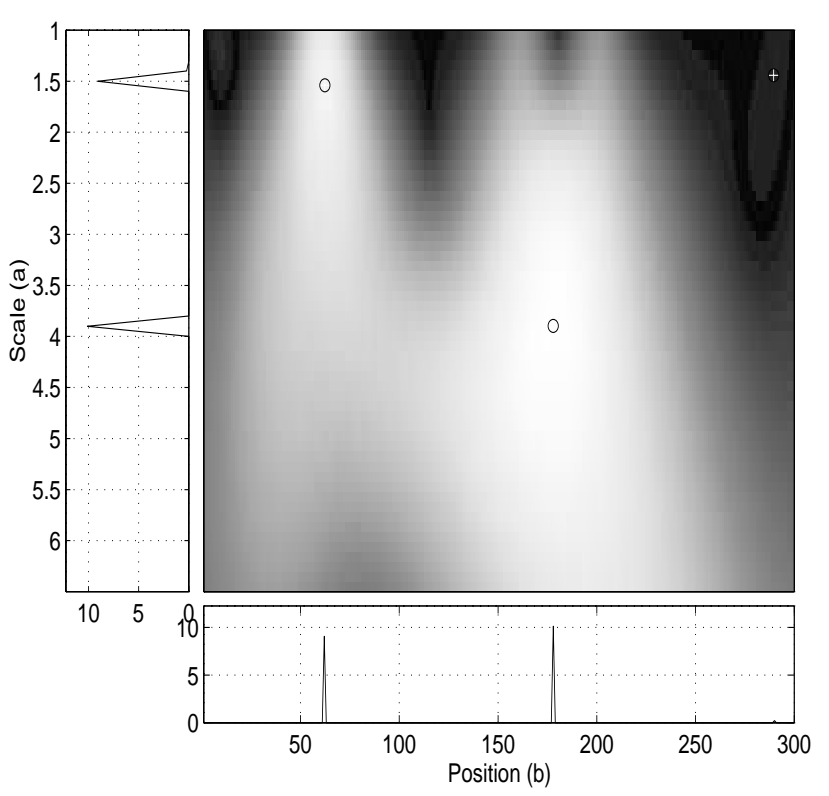

Figure 7. DWT of $\widetilde{O}(x)$

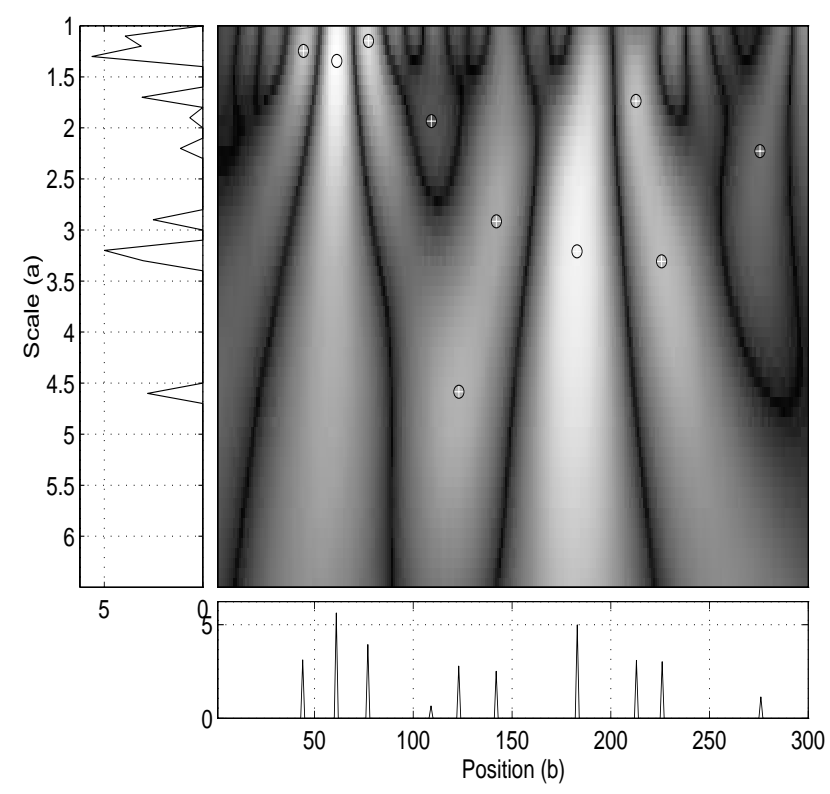

Figure 8. DWT of $D(x)$

[3] L. C. Vega, M. P. Cagigal, P. M. Prieto, "Determining the velocity of a moving object from a series of photonlimited frames using a temporal Fourier transform," SPIE conf. on signal data processing of small targets., pp.198-203, Orlando, FL, April 1993.

[4] G. Strang, T. Nguyen, Wavelets and Filter Banks, Wellesley, MA, USA: Wellesley-Cambridge Press, 1996.

[5] S. J. Schiff, "Resolving time-series structure with a controlled wavelet transform," Optical Engineering, pp. 2492-2495, Nov. 1992.

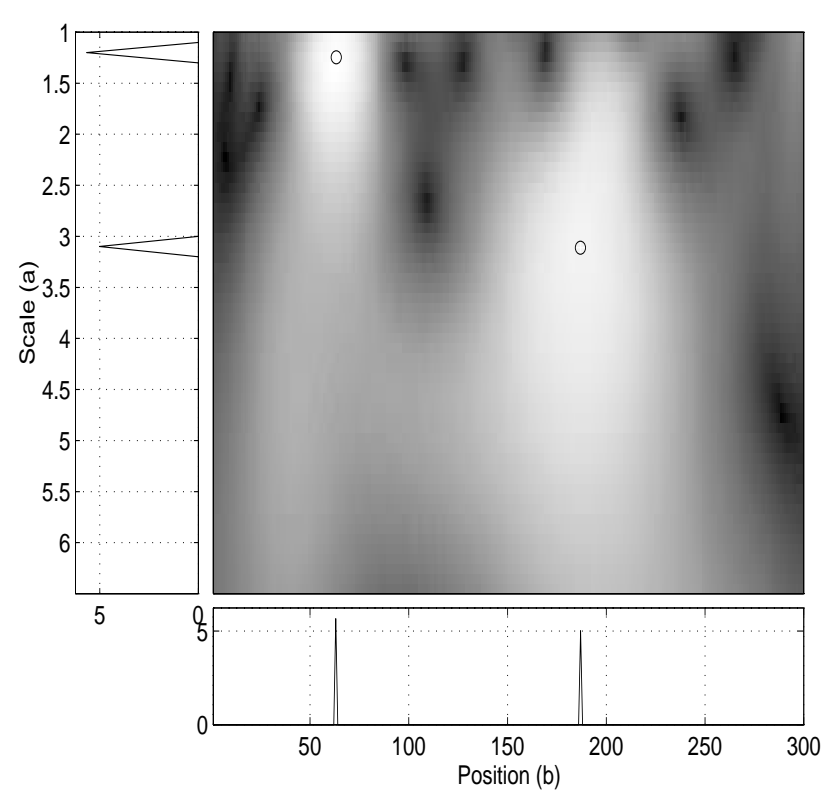

Figure 9. DWT of $\widetilde{D}(x)$

[6] M. Vetterli, J. Kovačević, Wavelets and Subband Coding, Englewood Cliffs, NJ: Prentice-Hall, 1995. 